\title{
CLINICAL USES OF 2,3-DIMERCAPTOPROPANOL (BAL). IV. PHARMACOLOGIC OBSERVATIONS ON BAL BY INTRA- MUSCULAR INJECTION IN MAN ${ }^{1}$
}

\author{
By WALTER MODELL, HARRY GOLD, AND McKEEN CATTELL \\ (From the Department of Pharmacology, Cornell University Medical College)
}

(Received for publication February 5, 1946)

The present report deals with a study ${ }^{2}$ of the effects of 2,3-dimercaptopropanol (BAL) in humans. It was designed to explore the range of doses which may be safely administered to humans in relation to the schedules of doses which have been found effective in the treatment of Lewisite poisoning in animals.

\section{SUBJECTS}

The experiments were carried out in 9 subjects, 8 females and 1 male, 7 colored and 2 white. Their ages ranged from 18 to 49 . They all had secondary or tertiary lues. One had diabetes and another hypertension, and a third, rheumatic heart disease. Three were under active antiluetic treatment; the remaining ones had not received treatment for at least several months.

\section{PLAN}

In each of 4 cases the experiments were carried out in the outpatient clinic, the patient leaving the clinic after symptoms had subsided. In each of 5 cases the patient was placed in the hospital, and was discharged on the day following the last dose. The experiment was so conducted as to eliminate anticipation or fear as a factor in the subjective symptoms.

American Reference Standard BAL was used in a 10 per cent solution in peanut oil containing 20 per cent benzyl benzoate (Hinson, Westcott and Dunning). It was injected deeply into the gluteal muscles.

In the general plan the subject was weighed and placed in bed or on an examining couch, where he remained throughout the experiment. The blood pressure, the heart rate, and in some cases

\footnotetext{
1 The work described in this paper was done under contract, recommended by the Committee on Medical Research, between the Office of Scientific Research and Development and the Cornell University Medical College.

2 The experiments were carried out at the Hospital for Joint Diseases, New York.
}

the respirations were recorded at approximately 10-minute intervals for 30 minutes prior to the injection, and at approximately 15-minute intervals for about an hour after the injection. Symptoms were recorded as they occurred until the effects had completely or nearly completely disappeared.

\section{RESULTS}

Dosage. The schedules of dosage and the results are summarized in Tables I and II. All doses were administered in terms of $\mathrm{mgm}$. per $\mathrm{kgm}$. of body weight. Single doses varied from 3.0 to $8.0 \mathrm{mgm}$. per $\mathrm{kgm}$.; and total amounts at each injection, from 133.5 to $640 \mathrm{mgm}$. (1.3 to $6.4 \mathrm{ml}$. of the 10 per cent solution). In those cases in which more than 1 injection was given in a day, the total doses varied from 8.0 to 20.0 mgm. per kgm. The latter amounts were administered in a period of from 8 to 10 hours.

In the dosage range employed in these experiments (3.0 to $5.0 \mathrm{mgm}$.) distinct cumulation occurred when doses were given at intervals of 30 minutes. The 30 -minute interval, however, greatly reduced the toxicity, since $8.0 \mathrm{mgm}$. per $\mathrm{kgm}$. at one time produced severe symptoms, while the same amount given in a dose of $5.0 \mathrm{mgm}$. and 3.0 mgm. with an interval of 30 minutes, produced relatively mild symptoms in the same person.

Cumulation also occurred when the second dose was given at an interval of 2 hours. It may be fairly marked, as shown by case $\mathrm{Li} \mathrm{Co}$, in which slight symptoms from the first dose of $5.0 \mathrm{mgm}$. became very severe symptoms when the same dose was repeated in 2 hours.

A 3-hour interval between doses seemed to permit negligible cumulation, although we had only 1 case (An Ma) on this schedule. With the 4 hour interval between doses there was no cumulation.

Effects. A single dose of $3.0 \mathrm{mgm}$. per $\mathrm{kgm}$. produced no symptoms. A single dose of $\mathbf{5 . 0}$ 
mgm. per $\mathrm{kgm}$. produced practically no symptoms in half of the cases (4 out of 8 ); and in the remaining ones, symptoms of moderate severity. After a single dose of $8.0 \mathrm{mgm}$. per $\mathrm{kgm}$. the symptoms were among the most severe.

Following the injection of BAL intramuscularly, symptoms appeared in from 5 to 30 minutes. They gradually developed in intensity, reaching a plateau which lasted about 30 minutes, and then subsided gradually. In some, the entire course of symptoms lasted only about 15 minutes, while in others the symptoms persisted for as long as 2 hours.

The heart rate was usually accelerated moder- ately during the peak of the symptoms. A rise of the blood pressure was one of the most regular effects. It was seen in 7 out of 9 cases. It was usually present after the second dose of $5.0 \mathrm{mgm}$., although occasionally after the first dose. Both the systolic and diastolic pressures rose. The increase ranged from 14 to $52 \mathrm{~mm}$. $\mathrm{Hg}$ systolic, and from 10 to $40 \mathrm{~mm}$. $\mathrm{Hg}$ diastolic. The course of the blood pressure change usually paralleled the symptoms. The only subject in whom the blood pressure failed to rise was the one with hypertension (An Mo). In this case the blood pressure fell. The patient started in the control period as a hypertensive_in the range of $180 / 90$, and

TABLE I

Effects of 10 per cent solution of BAL in various dosage schedules by intramuscular injection in man

\begin{tabular}{|c|c|c|c|c|c|c|c|c|c|c|}
\hline \multirow{3}{*}{ Case } & \multirow{3}{*}{ Date } & \multicolumn{2}{|c|}{ Dose of BAL } & \multirow{3}{*}{$\begin{array}{c}\text { Interval since } \\
\text { last dose }\end{array}$} & \multicolumn{3}{|c|}{ Effect on } & \multicolumn{3}{|c|}{ Symptoms } \\
\hline & & \multirow{2}{*}{ per kgm. } & \multirow{2}{*}{ Total } & & \multirow{2}{*}{ Heart rate } & \multicolumn{2}{|c|}{ B. P. (mm. Hg.) } & \multirow{2}{*}{$\begin{array}{l}\text { Intensity } \\
0 \text { to } 4+\end{array}$} & \multirow{2}{*}{ Onset } & \multirow{2}{*}{ Duration* } \\
\hline & & & & & & $\mathbf{S}$ & D & & & \\
\hline $\mathrm{Cl} \mathrm{Wa}$ & $\begin{array}{l}4 / 18 \\
4 / 21\end{array}$ & $\begin{array}{c}m g m . \\
3.0 \\
5.0 \\
5.0\end{array}$ & $\begin{array}{l}m g m . \\
177.3 \\
295.0 \\
295.0\end{array}$ & $30 \mathrm{~min}$. & $\begin{array}{r}0 \\
0 \\
+8\end{array}$ & $\begin{array}{r}0 \\
0 \\
+32\end{array}$ & $\begin{array}{r}0 \\
0 \\
+18\end{array}$ & $\begin{array}{r}0 \\
0 \\
++\end{array}$ & $\begin{array}{l}\min . \\
10\end{array}$ & $\begin{array}{l}\min . \\
43\end{array}$ \\
\hline $\mathrm{El} \mathrm{De}$ & $\begin{array}{l}4 / 20 \\
4 / 21\end{array}$ & $\begin{array}{l}5.0 \\
3.0 \\
8.0\end{array}$ & $\begin{array}{l}400.0 \\
240.0 \\
640.0\end{array}$ & $30 \mathrm{~min}$. & $\begin{array}{r}0 \\
0 \\
+30\end{array}$ & $\begin{array}{r}0 \\
+32 \\
+28\end{array}$ & $\begin{array}{r}0 \\
+38 \\
+14\end{array}$ & $+\stackrel{t}{+}+t$ & $\begin{array}{r}18 \\
8\end{array}$ & $\begin{array}{r}131 \\
52\end{array}$ \\
\hline Et Wo & $4 / 19$ & $\begin{array}{l}5.0 \\
3.0\end{array}$ & $\begin{array}{l}245.0 \\
141.0\end{array}$ & $32 \mathrm{~min}$. & $\begin{array}{r}+8 \\
+20\end{array}$ & $\begin{array}{r}0 \\
+30\end{array}$ & $\begin{array}{r}0 \\
+32\end{array}$ & $++t$ & $\begin{array}{r}25 \\
5\end{array}$ & $\begin{array}{l}30 \\
59\end{array}$ \\
\hline Ma Jo & $4 / 18$ & 3.0 & 133.5 & & 0 & 0 & 0 & 0 & & \\
\hline Id Vi & $4 / 29$ & $\begin{array}{l}5.0 \\
5.0 \\
5.0\end{array}$ & $\begin{array}{l}320.0 \\
320.0 \\
320.0\end{array}$ & $\begin{array}{l}2 \mathrm{hrs} . \\
4 \mathrm{hrs} .\end{array}$ & $\begin{array}{l}+14 \\
+24 \\
+24\end{array}$ & $\begin{array}{l}+26 \\
+32 \\
+30\end{array}$ & $\begin{array}{l}+20 \\
+36 \\
+24\end{array}$ & $\begin{array}{r}+ \\
++ \\
++\end{array}$ & $\begin{array}{l}30 \\
17 \\
15\end{array}$ & $\begin{array}{l}70 \\
40 \\
50\end{array}$ \\
\hline An Mo & $5 / 1$ & $\begin{array}{l}5.0 \\
5.0 \\
5.0 \\
5.0\end{array}$ & $\begin{array}{l}320.0 \\
320.0 \\
320.0 \\
320.0\end{array}$ & $\begin{array}{l}2 \text { hrs. } \\
4 \text { hrs. } \\
4 \text { hrs. }\end{array}$ & $\begin{array}{l}0 \\
0 \\
0 \\
0\end{array}$ & $\begin{array}{r}-12 \\
-44 \\
+8 \\
-16\end{array}$ & $\begin{array}{r}0 \\
-38 \\
+12 \\
0\end{array}$ & $\begin{array}{r}0 \\
++ \\
+ \\
0\end{array}$ & $\begin{array}{l}12 \\
30\end{array}$ & $\begin{array}{l}46 \\
21\end{array}$ \\
\hline Li Co & $5 / 3$ & $\begin{array}{l}5.0 \\
5.0 \\
5.0 \\
5.0\end{array}$ & $\begin{array}{l}300.0 \\
300.0 \\
300.0 \\
300.0\end{array}$ & $\begin{array}{l}2 \text { hrs. } \\
4 \text { hrs. } \\
4 \text { hrs. }\end{array}$ & $\begin{array}{r}+12 \\
0 \\
+12 \\
0\end{array}$ & $\begin{array}{r}+14 \\
+52 \\
+16 \\
0\end{array}$ & $\begin{array}{r}+20 \\
+40 \\
+12 \\
0\end{array}$ & $\begin{array}{r}++ \\
+ \\
\pm\end{array}$ & $\begin{array}{l}30 \\
15- \\
25 \\
14\end{array}$ & $\begin{array}{l}80- \\
29 \\
15\end{array}$ \\
\hline An $\mathrm{Ma}$ & $5 / 4$ & $\begin{array}{l}5.0 \\
5.0 \\
5.0 \\
5.0\end{array}$ & $\begin{array}{l}284.0 \\
284.0 \\
284.0 \\
284.0\end{array}$ & $\begin{array}{l}2 \text { hrs. } \\
3 \text { hrs. } \\
3 \text { hrs. }\end{array}$ & $\begin{array}{r}0 \\
0 \\
0 \\
-14\end{array}$ & $\begin{array}{l}+10 \\
+42 \\
+36 \\
-12\end{array}$ & $\begin{array}{r}+14 \\
+28 \\
+26 \\
-8\end{array}$ & $\begin{array}{l}0 \\
\pm \\
+ \\
0\end{array}$ & $\begin{array}{l}33 \\
15\end{array}$ & $\begin{array}{l}15 \\
30\end{array}$ \\
\hline Is $\mathrm{Bl}$ & $5 / 5$ & $\begin{array}{l}5.0 \\
5.0 \\
5.0 \\
5.0\end{array}$ & $\begin{array}{l}293.0 \\
293.0 \\
293.0 \\
293.0\end{array}$ & $\begin{array}{l}2 \text { hrs. } \\
4 \text { hrs. } \\
4 \text { hrs. }\end{array}$ & $\begin{array}{l}+24 \\
+24 \\
+16 \\
+28\end{array}$ & $\begin{array}{r}+14 \\
+40 \\
0 \\
+10\end{array}$ & $\begin{array}{l}+38 \\
+28 \\
+14 \\
+10\end{array}$ & $\begin{array}{r}++ \\
++t \\
+\end{array}$ & $\begin{array}{r}17 \\
7 \\
16 \\
26\end{array}$ & $\begin{array}{l}27 \\
37 \\
50 \\
28\end{array}$ \\
\hline
\end{tabular}

* In most instances mild and vague symptoms continued after the last observation and wore off gradually.

S (systolic); D (diastolic). 
TABLE II

Symptoms produced by $B A L$ in man

\begin{tabular}{|c|c|c|c|c|c|c|c|c|c|c|c|c|c|}
\hline \multirow[b]{2}{*}{ Case } & \multirow[b]{2}{*}{ Date } & \multirow{2}{*}{$\begin{array}{l}\text { Dose } \\
\text { of } \\
\text { BAL }\end{array}$} & \multirow{2}{*}{$\begin{array}{c}\text { Interval } \\
\text { since last } \\
\text { dose }\end{array}$} & \multicolumn{10}{|c|}{ Symptoms } \\
\hline & & & & Nausea & $\begin{array}{l}\text { Vom- } \\
\text { iting }\end{array}$ & Salivation & Eye & Pain & Anxiety & Unrest & $\begin{array}{c}\text { Paresthe- } \\
\text { sias }\end{array}$ & $\left|\begin{array}{l}\text { Local } \\
\text { pain }\end{array}\right|$ & Warmth \\
\hline $\mathrm{Cl} \mathrm{Wa}$ & $\begin{array}{l}4 / 18 \\
4 / 21\end{array}$ & 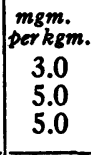 & $\cdot$ & $\begin{array}{l}0 \\
0 \\
0\end{array}$ & $\begin{array}{l}0 \\
0 \\
0\end{array}$ & $\begin{array}{l}0 \\
0 \\
0\end{array}$ & $\begin{array}{r}0 \\
0 \\
+\end{array}$ & $\begin{array}{r}0 \\
0 \\
++\end{array}$ & $\begin{array}{r}0 \\
0 \\
+\end{array}$ & $\begin{array}{r}0 \\
0 \\
++\end{array}$ & $\begin{array}{r}0 \\
0 \\
+\end{array}$ & $\begin{array}{l}0 \\
0 \\
0\end{array}$ & $\begin{array}{l}0 \\
0 \\
0\end{array}$ \\
\hline El De & $\begin{array}{l}4 / 20 \\
4 / 21\end{array}$ & $\begin{array}{l}5.0 \\
3.0 \\
8.0\end{array}$ & $30 \mathrm{~min}$. & $\begin{array}{r} \pm \\
+ \\
+\end{array}$ & $\begin{array}{r}0 \\
0 \\
+\end{array}$ & $\begin{array}{l}0 \\
+ \\
+\end{array}$ & $\begin{array}{l}0 \\
0 \\
0\end{array}$ & $\begin{array}{r}0 \\
+++\end{array}$ & $\begin{array}{r}0 \\
0 \\
++++\end{array}$ & $\begin{array}{r}0 \\
+++\end{array}$ & $\begin{array}{l}0 \\
+ \\
+\end{array}$ & $\begin{array}{r}0 \\
+ \\
0\end{array}$ & $\begin{array}{l}0 \\
+ \\
0\end{array}$ \\
\hline Et Wo & $4 / 19$ & $\begin{array}{l}5.0 \\
3.0\end{array}$ & $32 \mathrm{~min}$. & $\begin{array}{l}0 \\
0\end{array}$ & $\begin{array}{l}0 \\
0\end{array}$ & $++++^{0}$ & $\begin{array}{l}0 \\
0\end{array}$ & $\stackrel{0}{+}$ & $+++\stackrel{0}{+}$ & $++++^{0}$ & + & + & $\begin{array}{l}0 \\
+\end{array}$ \\
\hline Ma Jo & $4 / 18$ & 3.0 & & 0 & 0 & 0 & 0 & 0 & 0 & 0 & 0 & 0 & 0 \\
\hline Id $\mathrm{Vi}$ & $4 / 29$ & $\begin{array}{l}5.0 \\
5.0 \\
5.0\end{array}$ & $\begin{array}{l}2 \text { hrs. } \\
4 \text { hrs. }\end{array}$ & $\begin{array}{l}+ \\
+ \\
+\end{array}$ & $\begin{array}{l}++ \\
+ \\
++\end{array}$ & ++ & $\begin{array}{l}0 \\
0 \\
0\end{array}$ & $\begin{array}{r}++ \\
+++ \\
+++\end{array}$ & $\begin{array}{l}++ \\
+ \\
+\end{array}$ & $\mid \begin{array}{r}++ \\
+++ \\
+++\end{array}$ & $+++\underset{0}{+}$ & $\begin{array}{l}0 \\
+ \\
+\end{array}$ & $\begin{array}{l}0 \\
0 \\
0\end{array}$ \\
\hline An Mo & $5 / 1$ & $\begin{array}{l}5.0 \\
5.0 \\
5.0 \\
5.0\end{array}$ & $\begin{array}{l}2 \text { hrs. } \\
4 \text { hrs. } \\
4 \text { hrs. }\end{array}$ & $\begin{array}{l}0 \\
0 \\
0 \\
0\end{array}$ & $\begin{array}{l}0 \\
0 \\
0 \\
0\end{array}$ & $\begin{array}{r}0 \\
++ \\
+ \\
0\end{array}$ & $\begin{array}{l}0 \\
0 \\
0 \\
0\end{array}$ & $\begin{array}{l}0 \\
0 \\
0 \\
0\end{array}$ & $\begin{array}{l}0 \\
0 \\
0 \\
0\end{array}$ & $\begin{array}{l}0 \\
0 \\
0 \\
0\end{array}$ & $\begin{array}{r}0 \\
++ \\
+ \\
0\end{array}$ & $\begin{array}{l}\mathbf{0} \\
\mathbf{0} \\
\mathbf{0} \\
\mathbf{0}\end{array}$ & $\begin{array}{l}\mathbf{0} \\
\mathbf{0} \\
\mathbf{0} \\
\mathbf{0}\end{array}$ \\
\hline Li Co & $5 / 3$ & $\begin{array}{l}5.0 \\
5.0 \\
5.0 \\
5.0\end{array}$ & $\begin{array}{l}2 \text { hrs. } \\
4 \text { hrs. } \\
4 \text { hrs. }\end{array}$ & $+++\underset{0}{0}$ & $\begin{array}{l}0 \\
0 \\
0 \\
0\end{array}$ & $\begin{array}{l}+ \\
+ \\
0 \\
+\end{array}$ & $\begin{array}{l}0 \\
0 \\
0 \\
0\end{array}$ & $+++\frac{+}{+}+$ & $\begin{array}{l}0 \\
0 \\
0 \\
0\end{array}$ & $\begin{array}{r}0 \\
+++ \\
0 \\
0\end{array}$ & $\begin{array}{r}0 \\
+ \\
0 \\
0\end{array}$ & $\begin{array}{r}+ \\
0 \\
0 \\
0\end{array}$ & $\begin{array}{l}0 \\
0 \\
0 \\
0\end{array}$ \\
\hline An $\mathrm{Ma}$ & $5 / 4$ & $\begin{array}{l}5.0 \\
5.0 \\
5.0 \\
5.0\end{array}$ & $\begin{array}{l}2 \text { hrs. } \\
3 \text { hrs. } \\
3 \text { hrs. }\end{array}$ & $\begin{array}{l}0 \\
0 \\
0 \\
0\end{array}$ & $\begin{array}{l}0 \\
0 \\
0 \\
0\end{array}$ & $\begin{array}{l}0 \\
0 \\
0 \\
0\end{array}$ & $\begin{array}{l}0 \\
0 \\
0 \\
0\end{array}$ & $\begin{array}{l}0 \\
0 \\
+ \\
0\end{array}$ & $\begin{array}{l}\mathbf{0} \\
\mathbf{0} \\
\mathbf{0} \\
\mathbf{0}\end{array}$ & $\begin{array}{l}0 \\
0 \\
0 \\
0\end{array}$ & $\begin{array}{l}0 \\
\pm \\
+ \\
0\end{array}$ & $\begin{array}{l}0 \\
0 \\
0 \\
0\end{array}$ & $\begin{array}{l}0 \\
0 \\
+ \\
0\end{array}$ \\
\hline Is $\mathrm{Bl}$ & $5 / 5$ & $\begin{array}{l}5.0 \\
5.0 \\
5.0 \\
5.0\end{array}$ & $\begin{array}{l}2 \text { hrs. } \\
4 \text { hrs. } \\
4 \text { hrs. }\end{array}$ & $\begin{array}{l}+ \\
+ \\
+\end{array}$ & $\begin{array}{l}+ \\
+ \\
+ \\
+\end{array}$ & $\begin{array}{l}++ \\
++ \\
+ \\
+\end{array}$ & $\begin{array}{r}0 \\
++ \\
0 \\
0\end{array}$ & $\begin{array}{r}++ \\
0 \\
0 \\
0\end{array}$ & $\begin{array}{l}0 \\
0 \\
0 \\
0\end{array}$ & $\begin{array}{l}0 \\
0 \\
0 \\
0\end{array}$ & $\begin{array}{r}++ \\
++\underset{0}{+} \\
+\underset{0}{+}\end{array}$ & $\begin{array}{l}++ \\
++ \\
++ \\
++\end{array}$ & $\begin{array}{l}0 \\
+ \\
0 \\
0\end{array}$ \\
\hline
\end{tabular}

ended after the fourth $5.0 \mathrm{mgm}$. dose of BAL in 10 hours in the range of $138 / 70$. There were no symptoms associated with this fall in the blood pressure.

There is some indication of the development of tolerance to BAL since, as may be seen in Table II, the fourth, and sometimes the third, dose of $5.0 \mathrm{mgm}$. produced less severe symptoms than the first.

Symptoms. The pattern of symptoms varied from case to case (see Table II). Vomiting was usually associated with the most severe reactions. In the course of the more severe reactions, the patients showed paresthesias (burning or tingling of the nose, eyes, mouth or skin), pain (involv- ing the limbs, jaws, abdomen, chest and head), lacrimation, blepharospasm, and salivation. An indescribable sense of illness developed with extreme unrest and apprehension. Occasionally the patient perspired freely and complained of being very warm. As the symptoms subsided, most patients with the more severe reactions complained of weakness and fatigue. At 4 hours after the $\mathbf{5 . 0}$ $\mathrm{mgm}$. doses they were free of systemic symptoms.

While most doses caused some pain during the injection, a moderately tender area in the region of the injection, still present some hours later, was encountered in 4 of the 9 cases.

The complete protocols of the experiments are appended. 
Schedules. Single doses of BAL of from 5 to $8 \mathrm{mgm}$. per $\mathrm{kgm}$. intramuscularly in man produced reactions which did not appear to be dangerous, so also for total doses of $20 \mathrm{mgm}$. per $\mathrm{kgm}$. in fractions of $5 \mathrm{mgm}$. over a period of 8 hours.

A single dose of $5 \mathrm{mgm}$. per $\mathrm{kgm}$. caused no symptoms in 50 per cent of the cases, negligible effects in 25 per cent, and slight and fleeting symptoms in the remaining 25 per cent. The course of the reaction is fairly brief, lasting approximately an hour. The indications are that a schedule of dosage involving $5 \mathrm{mgm}$. per $\mathrm{kgm}$. for 4 doses at intervals of 3 hours is likely to show little cumulation. In such a schedule, the effects of the first dose are not likely to become greater as a result of its repetition. The effect of the third and fourth dose may actually be less. Shorter intervals with such doses, namely 2 hours or less, result in cumulation.

The review by Waters and Stock (1) indicates that others have obtained substantially similar results $(2,3)$.

\section{SUMMARY AND CONCLUSIONS}

1. The toxic effects of BAL by intramuscular injection were studied in 9 human subjects with secondary or tertiary lues.

2. The results are based on 28 injections of single doses of from 3 to $8 \mathrm{mgm}$. per $\mathrm{kgm}$.

3. In man BAL produces paresthesias (burning or tingling of the nose, eyes, mouth, and skin), perspiration and sense of warmth, pain (limbs, jaws, abdomen, head), lacrimation, blepharospasm, salivation, vomiting, unrest, apprehension, weakness, and fatigue. The heart rate is accelerated and both systolic and diastolic blood pressure usually increased.

4. The minimal dose which produces toxic effects lies between 3 and $5 \mathrm{mgm}$. per $\mathrm{kgm}$. A single dose of $8 \mathrm{mgm}$. produces marked symptoms.

5. The effects of doses up to $8 \mathrm{mgm}$. per $\mathrm{kgm}$. are completely reversible, the reactions lasting only about an hour or two.

6. Doses of $5 \mathrm{mgm}$. per $\mathrm{kgm}$. may be given at intervals of three hours during the course of one day without significant cumulation.

PROTOCOLS

Patient: $\mathrm{Cl} \mathrm{Wa}$; female; colored; age 18; wt. $59.1 \mathrm{kgm}$.

Lues; active treatment.

\section{$4 / 18 / 44$}

$\begin{array}{llcc}\text { Time } & \begin{array}{c}\text { Blood } \\ \text { pressure }\end{array} & \begin{array}{c}\text { Heart- } \\ \text { rate }\end{array} & \begin{array}{c}\text { Respir- } \\ \text { ation }\end{array} \\ 10: 40 & 110 / 74 & 108 & 22 \\ 10: 48 & 110 / 76 & 104 & 22 \\ 10: 55 & 106 / 72 & 98 & 22\end{array}$

$10: 55 \quad 106 / 72 \quad 98 \quad 22$

10:57 BAL, $3 \mathrm{mgm}$. per kgm. (1.8 ml.), muscle, local pain lasting $1 \mathrm{~min}$.

11:05 $\quad 108 / 78 \quad 104 \quad 24$

$11: 07 \quad 106 / 74 \quad 102 \quad 20$

$11: 18 \quad 108 / 76 \quad 100 \quad 21$

$11: 28 \quad 108 / 78 \quad 96 \quad 22$

$\begin{array}{llll}11: 42 & 104 / 72 & 92 & 24\end{array}$

$4 / 21 / 44$

$1: 46$

$1: 57$

2:16

$2: 25$

$2: 38$

$2: 48$

$2: 55$

$2: 56$

3:05

$3: 10$

$3: 20$

$3: 38$

$3: 48$

$\begin{array}{cc}116 / 74 & 76 \\ 120 / 68 & 74 \\ 116 / 80 & 88 \\ \text { BAL, } 5 \text { mgm. per kgm. } & (2.95 \\ 110 / 74 & 88 \\ 106 / 72 & 86 \\ \text { BAL, second dose as above, left } & 84 \\ 118 / 78 & 96 \\ 126 / 84 & \\ 152 / 98 & 96 \\ 130 / 90 & 94 \\ 128 / 80 & 80 \\ 116 / 76 & 76\end{array}$

$95 \mathrm{ml}$.$) , right gluteus, no local pain.$

86
84

4
Remarks-Symptoms

No complaints offered at any time, "I feel fine except my hip is stiff from needle," fell asleep during observations.
"Feel fine except for medicine in my mouth," "pepper in eyes and mouth."

About the same.

Seems weak, restless, says chest hurts, cries.

Better, can hardly keep eyes open because of smarting.

Better, eyes almost normal. 
Patient: El De; female; colored; age 42; wt. 80 kgm.

$4 / 20 / 44$

Lues; no treatment.

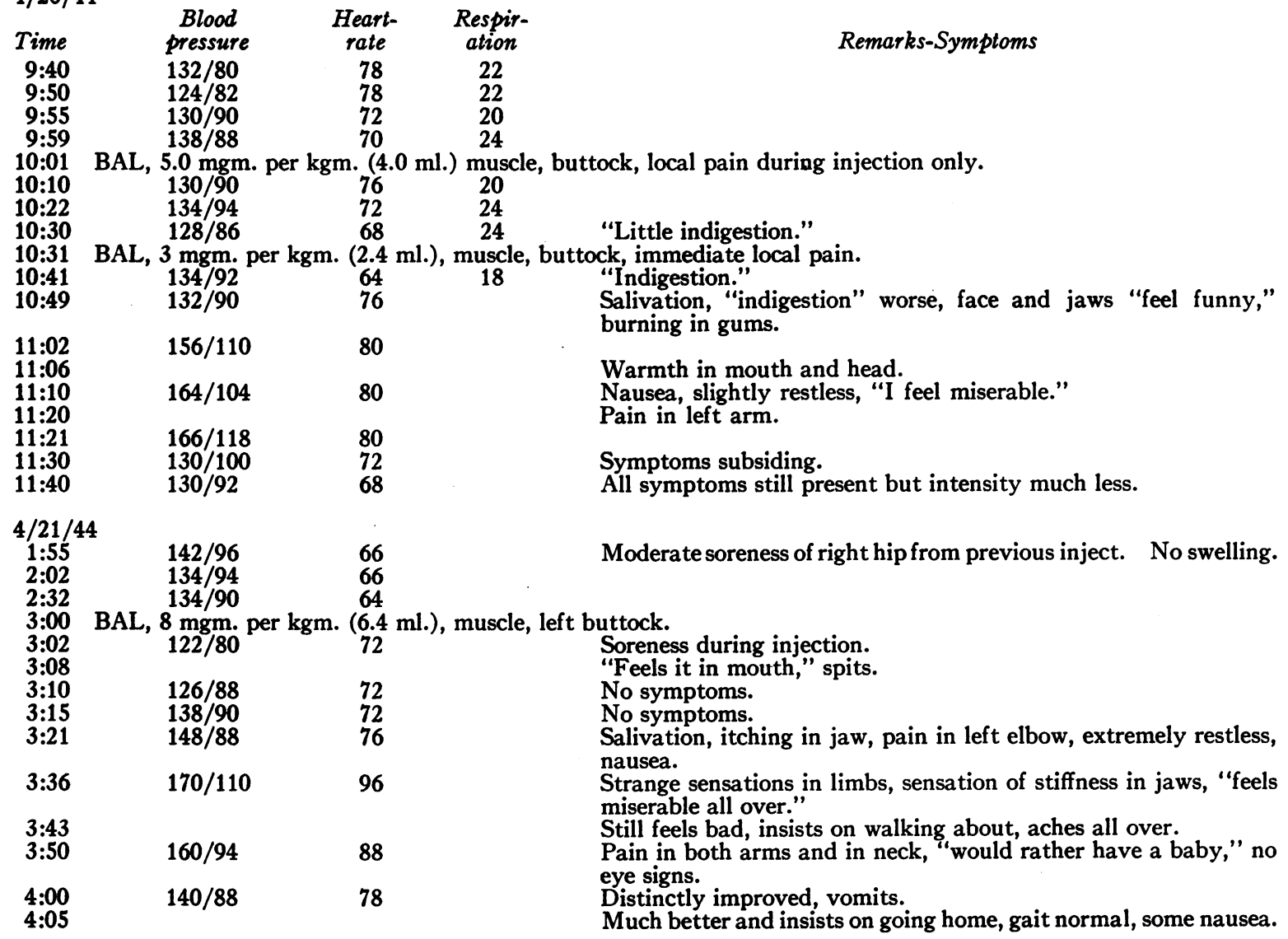

Patient: Et Wo; female; colored; age 26; wt. 49 kgm. $4 / 19 / 44$

Lues; active treatment.

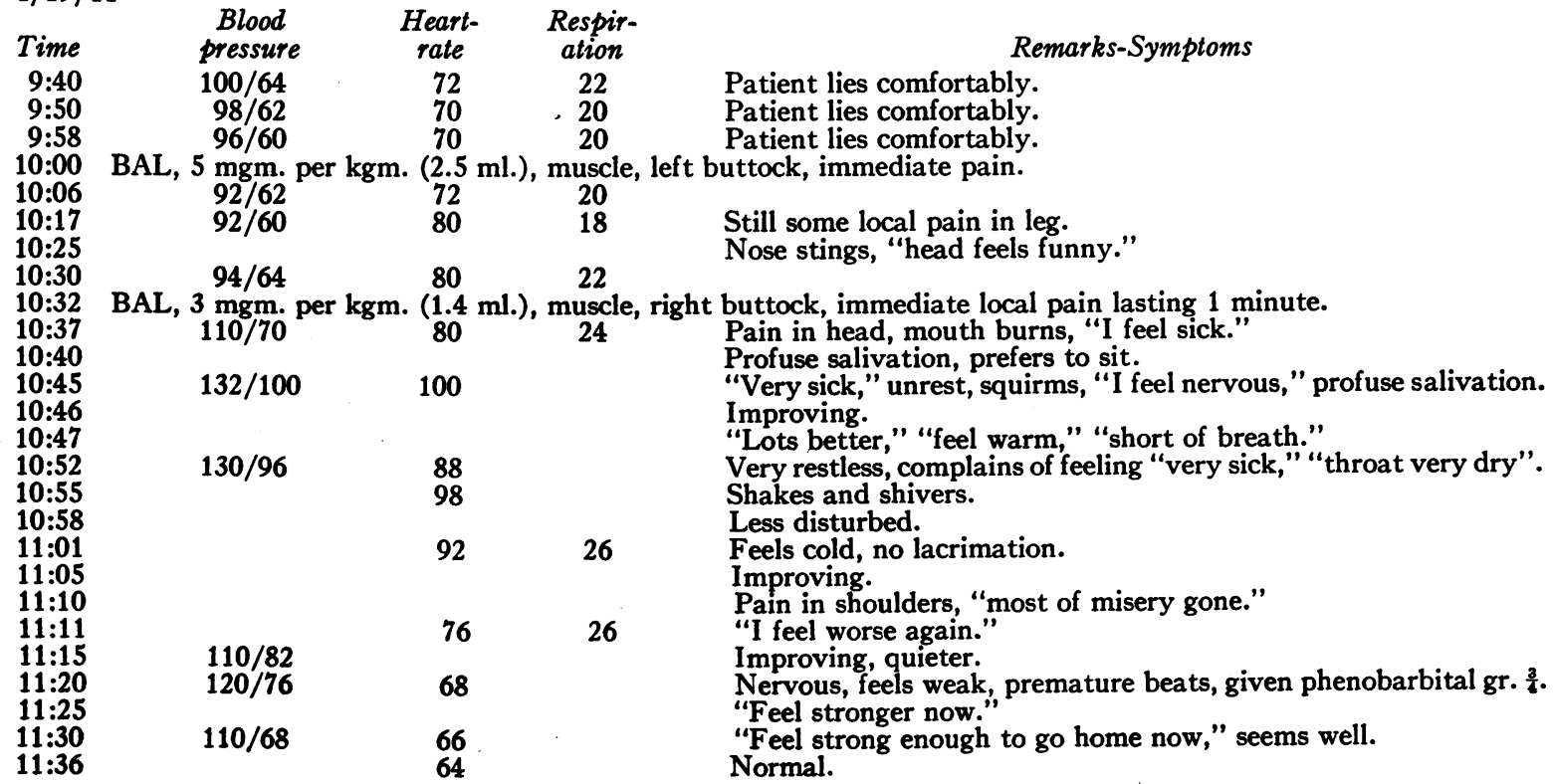


Patient: Ma Jo; female; colored; age 25; wt. $44.5 \mathrm{kgm}$.

Lues; active treatment; rheumatic heart disease. $4 / 18 / 44$

$\begin{array}{rcccl}\text { Time } & \begin{array}{c}\text { Blood } \\ \text { pressure }\end{array} & \begin{array}{c}\text { Heart- } \\ \text { rate }\end{array} & \begin{array}{c}\text { Respir- } \\ \text { ation }\end{array} & \text { Remarks-Symptoms } \\ \text { 9:34 } & 124 / 84 & 76 & 19 & \text { Patient lies comfortably. } \\ \text { 9:40 } & 130 / 82 & 76 & 16 & \text { Patient lies comfortably. } \\ \text { 9:48 } & 126 / 84 & 76 & 16 & \text { Patient lies comfortably. } \\ \text { 9:59 } & \text { BAL, } 3 \text { mgm. per kgm. }(1.3 \mathrm{ml} \text {.), } & \text { muscle, buttock, no local pain. } \\ \text { 10:04 } & 124 / 82 & 82 & 18 & \\ 10: 11 & 130 / 86 & 88 & 17 & \\ 10: 21 & 126 / 86 & 84 & 18 & \\ 10: 28 & 130 / 84 & 78 & 18 & \\ 10: 43 & 122 / 82 & 81 & 16 & \text { No complaints at any time, "I feel excellent." } \\ 11: 00 & 122 / 82 & 78 & 16 & \text {. }\end{array}$

Patient: Id Vi; female; white; age 34 ; wt. $64.5 \mathrm{kgm}$.

$4 / 29 / 44$

Lues; no treatment.

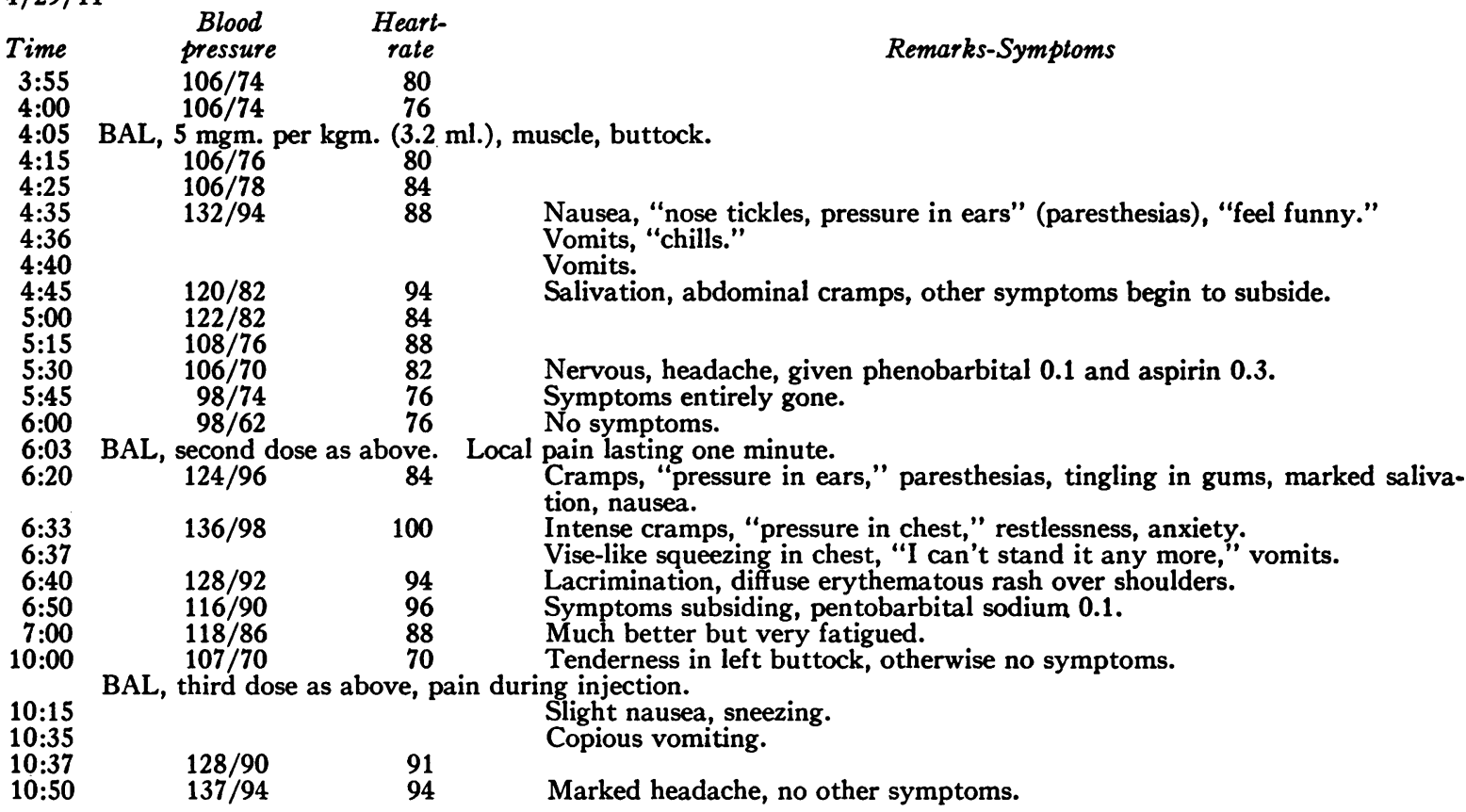

Patient: An Mo; female; colored; age 49; wt. $64.2 \mathrm{kgm}$.

$5 / 1 / 44$

Lues; no treatment.

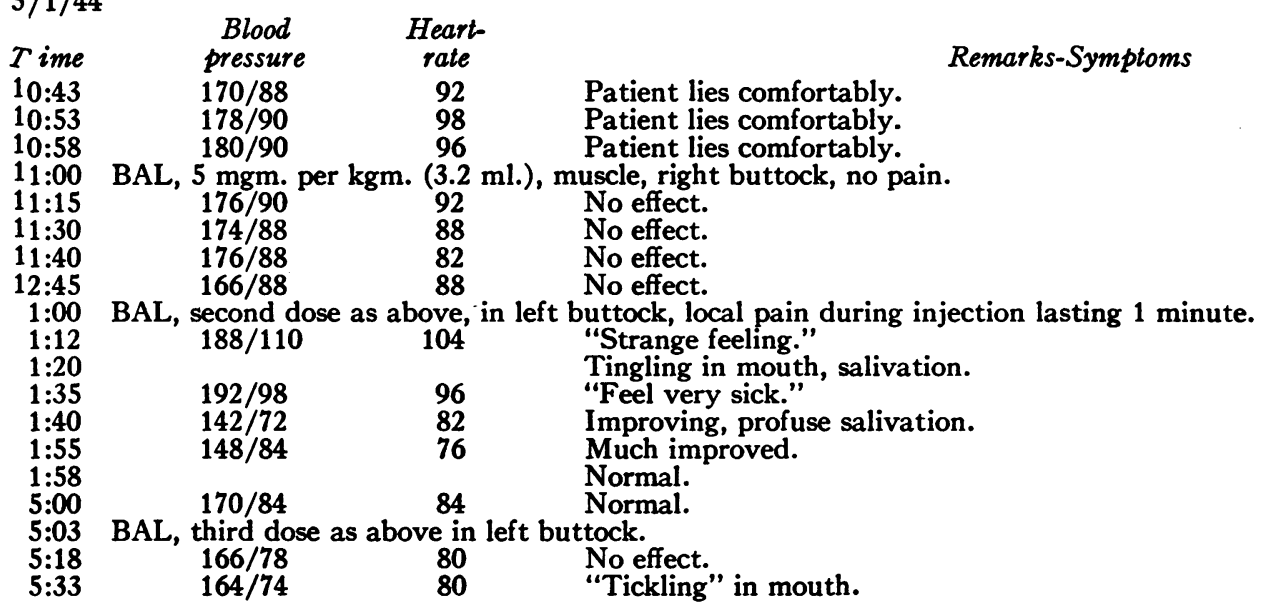




$\begin{array}{cccc}\text { 5:44 } & 174 / 90 & 84 & \begin{array}{c}\text { Itching in mouth, salivation. } \\ \text { 5:54 }\end{array} \\ \text { 8:46 } & 158 / 78 & 78 & \text { Normal. } \\ 8: 54 & \text { BAL, fourth dose as above in right } & \text { Normal. } \\ 9: 20 & 152 / 74 & 76 & \text { No effect. } \\ 9: 35 & 138 / 70 & 76 & \text { No effect. } \\ 9: 45 & 136 / 72 & 72 & \text { No effect. }\end{array}$

Patient: Li Co; female; colored; age 43 ; wt. 60 kgm. $5 / 3 / 44$

Lues, no treatment; diabetes; receives insulin.

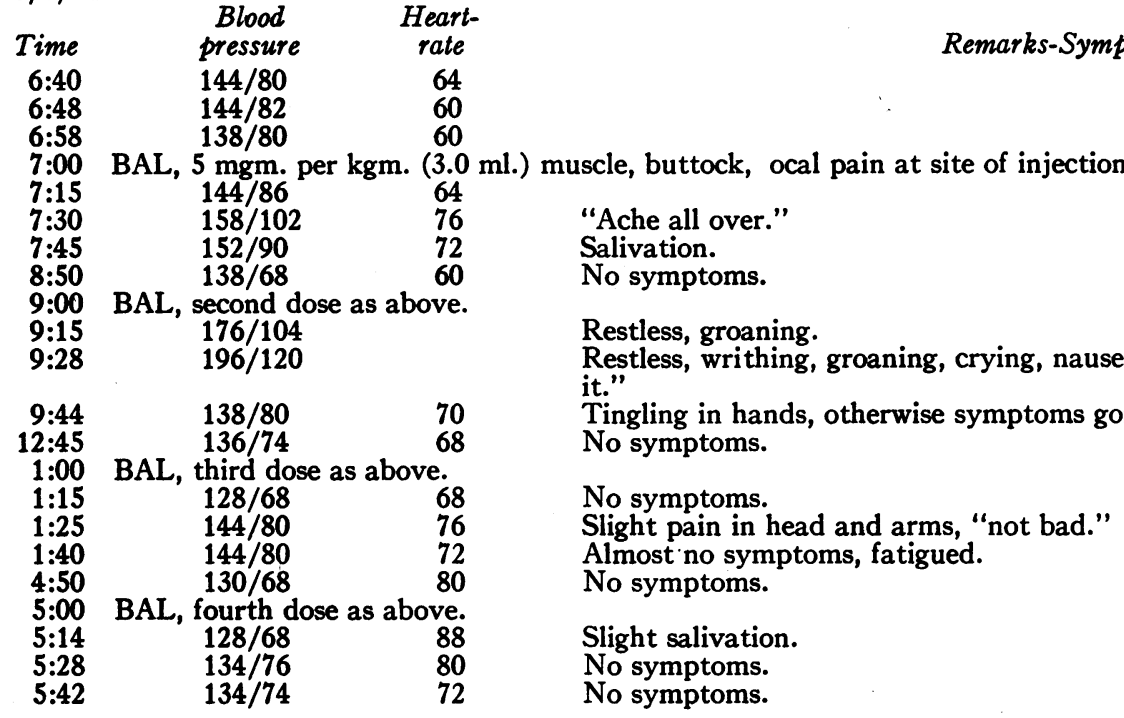

Patient: An Ma; white male; age 41 ; wt. $56.8 \mathrm{kgm}$. $5 / 4 / 44$

Lues; no treatment.

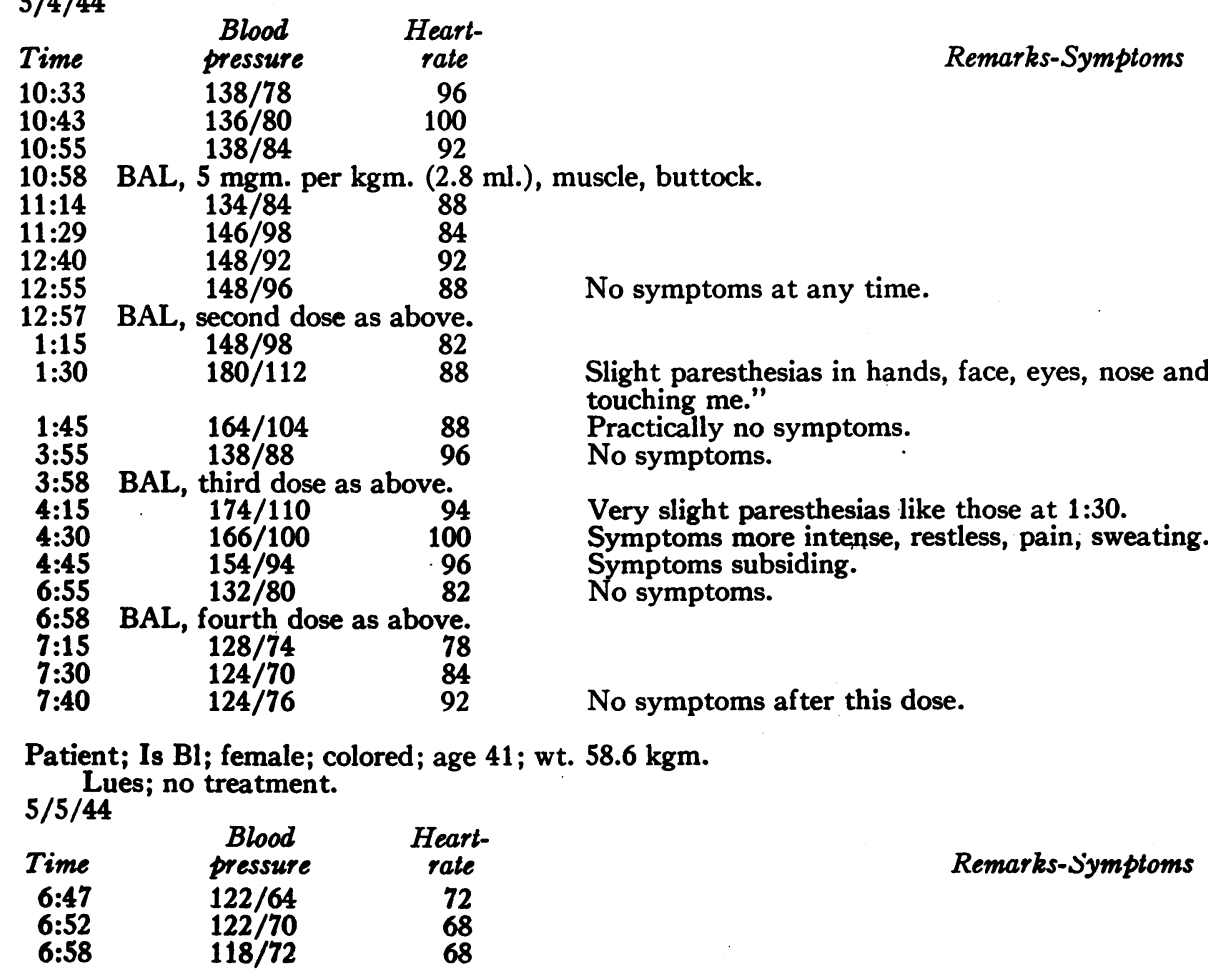


BAL, 5 mgm. per kgm. (2.9 ml.), muscle, left buttock. $132 / 80$ "Feel funny and sick all over," salivating.

130/110 $84 \quad$ Headache, vomited, perfuse salivation, pain in injected leg.

122/88 $92 \quad$ Better but still feeling sick.

132/84 96 No symptoms.

$\mathrm{BAL}$, second dose as above in right buttock.

"buttock.

154/98 $92 \quad$ "Hot," symptoms more severe than after first dose.

162/100 $96 \quad$ Lacrimination, symptoms slightly less.

$154 / 80 \quad 88 \quad$ Vomited, but otherwise better.

$118 / 70 \quad 88$ Hungry, no symptoms except pain in both buttocks.

BAL, third dose as above in left buttock.

$116 / 70$ "Funny feeling" starts, salivation.

$120 / 86 \quad 88 \quad$ Symptoms slightly more intense.

$116 / 72 \quad 84 \quad$ Nausea and vomiting, symptoms subsiding.

$128 / 70 \quad 98$

No symptoms, effects slightly less than from previous doses.

BAL, fourth dose as above in right buttock.

$\begin{array}{lcl}120 / 70 & 100 & \text { No effect. } \\ 124 / 72 & 96 & \text { "Feel something coming on," salivation. }\end{array}$

134/82 $98 \quad$ Feels better.

128/74 88 No symptoms other than pain at the site of injections, last dose caused least symptoms.

\section{BIBLIOGRAPHY}

1. Waters, L. L., and Stock, C. C., BAL (British AntiLewisite). Science, 1945, 102, 601.

2. Eagle, H., Magnuson, H. J., and Fleischman, R., The systemic treatment of experimental arsenic poisoning with BAL. J. Clin. Invest., 1946, 25, 451.

3. Sulzberger, M. B., Baer, R. L., and Kanof, A., Studies on the toxicity of BAL on percutaneous and parenteral administration. J. Clin. Invest., 1946, 25, 474. 